\title{
Outcomes of sleeve gastrectomy in patients older than 60 years: a multicenter matched case-control study
}

\author{
Natalia Dowgiałło-Wnukiewicz ${ }^{1}$, Michal R. Janik², Pawel Lech¹, Piotr Major ${ }^{3,4}$, Michał Pędziwiatr ${ }^{3,4}$, \\ Piotr K. Kowalewski², Maciej Walędziak², Michał Wysocki³,4, Maciej Michalik ${ }^{1}$ \\ ${ }^{1}$ Department of General, Minimally Invasive and Elderly Surgery, University of Warmia and Mazury, Olsztyn, Poland \\ ${ }^{2}$ Department of General, Oncologic, Metabolic and Thoracic Surgery, Military Institute of Medicine, Warsaw, Poland \\ ${ }^{3} 2^{\text {nd }}$ Department of General Surgery, Jagiellonian University Medical College, Krakow, Poland \\ ${ }^{4}$ Centre for Research, Training and Innovation in Surgery (CERTAIN Surgery), Krakow, Poland
}

Videosurgery Miniinv 2020; 15 (1): 123-128 DOI: https://doi.org/10.5114/wiitm.2019.81450

\begin{abstract}
Introduction: The prevalence of obesity is increasing according to the World Health Organization. Furthermore, global aging is increasing, especially in developed countries in Europe. Whether bariatric surgery should be performed in elderly people is still controversial.

Aim: To determine the clinical outcomes of sleeve gastrectomies (SG) in older central European patients. We compared the safety and efficacy of SG in patients older than 60 years with younger patients.

Material and methods: Eighty-nine patients older than 60 years, who underwent SG, were included in the study. Eighty-nine younger patients (aged 18-40 years) were matched according to body mass index (BMI) and comorbidities. The analyzed data included age, sex, total body weight, BMI, length of hospital stay, 30-day complications and improvement in comorbidities.

Results: There was no significant difference in the complication rate between the 2 age groups $(p=0.59)$. An improvement in hypertension was observed in $73.1 \%$ of older patients and in $69.2 \%$ of younger patients $(p=0.67)$. There was improvement in diabetes mellitus in 40\% of older patients and in $31.1 \%$ of younger patients $(p=0.25)$. The $\triangle B M I$ after 12, 24 and 36 months was significantly lower in older patients than in younger patients $(p=0.002$, $p=0.001 ; p=0.043$, respectively). Percent excess BMI loss (\%EBMIL) after 12, 24, and 60 months was significantly lower in older than in younger patients ( $p=0.001, p=0.001, p=0.028$, respectively).

Conclusions: Better weight loss is achieved in younger than in older patients, while maintaining a similar effect on the risk of complications and improvement in comorbidities. Therefore, SG is safe and effective in older people.
\end{abstract}

Key words: older age, elderly, sleeve gastrectomy, bariatric surgery.

\section{Introduction}

The prevalence of obesity in Europe and other parts of the world is increasing according to the World Health Organization [1]. Furthermore, global aging is increasing, especially in developed countries in Europe and the United States [2]. Consequently, an increasing number of older people will need treatment for obesity, as well as its comorbidities. Conservative methods, such as lifestyle modification, have poor long-term results for obesity. Therefore, bariatric surgery, which is an effective and durable treatment, is performed worldwide [3, 4]. Currently, sleeve gastrectomy (SG) is one of the most com-

\section{Address for correspondence}

Natalia Dowgiałło-Wnukiewicz MD, Department of General, Minimally Invasive and Elderly Surgery, University of Warmia and Mazury,

2 Michała Oczapowskiego St, 10-719 Olsztyn, Poland, phone: +48 604158 786, e-mail: natalia.dowgiallo@gmail.com 
Enrollment

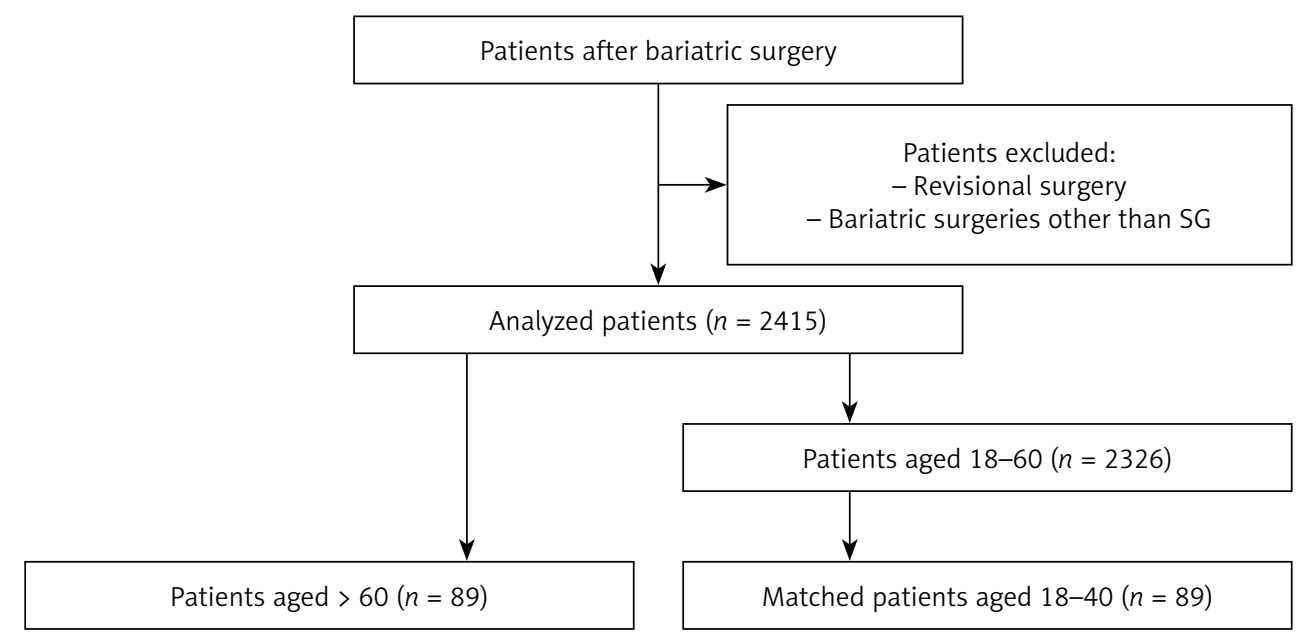

Allocation

Analysis$$
\text { ched patients aged } 18-40(n=89)
$$

Figure 1. Flow chart of the study

mon bariatric procedures [5]. Restriction in gastric volume and changes in secreted hormones lead to satisfactory weight loss and resolution or at least an improvement in comorbidities.

Whether surgery should be performed in elderly people is still controversial. Older age causes difficulties in surgery and is related to an increase in perioperative complications and mortality [6]. Qualification of elderly people for bariatric surgery should be made by evaluating the risks and benefits. Nevertheless, the number of performed procedures among older patients is increasing $[7,8]$. Complications, baseline general health status and life expectancy are taken into account when surgery is being considered. Recent reports have shown that bariatric surgery appears to be an effective therapy for obesity even in elderly people, especially because obesity is related to comorbidities such as diabetes mellitus or hypertension [9-14].

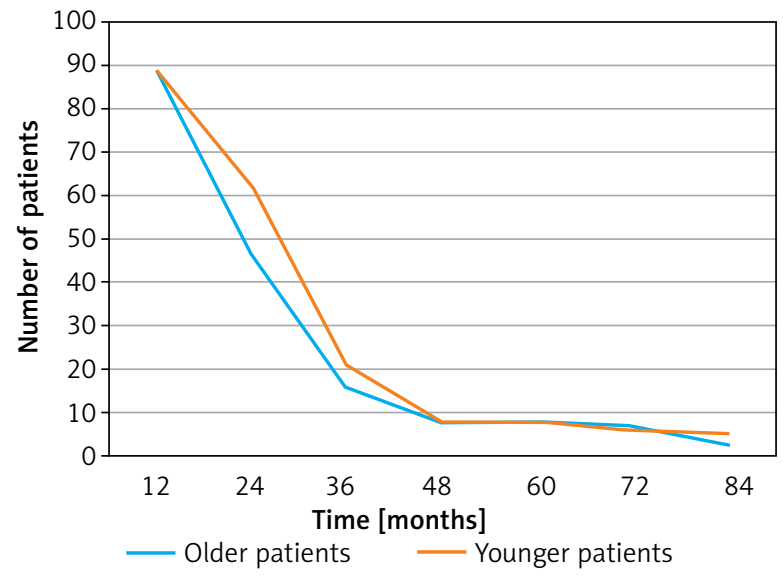

Figure 2. Number of patients during follow-up

\section{Aim}

This study aimed to determine the clinical outcomes of SG in older central European patients. We compared the safety and efficacy of SG in patients older than 60 years with younger patients.

\section{Material and methods}

A case-control study was performed in patients who underwent SG. Patients underwent surgery from 2009 to 2017 at four Polish high-volume bariatric centers. Eighty-nine patients were older than 60 years among 2415 patients who underwent surgery at this time. Eighty-nine patients were included as matched controls (aged 18-40 years) (Figure 1). All of the procedures were performed using the same surgical technique as described previously [15].

\section{Cases and controls}

Patients were followed up at 12 months and every year after surgery (Figure 2). The data that were analyzed included age, sex, total body weight and body mass index (BMI), length of stay in hospital (LOS), and complications. We also analyzed comorbidities such as diabetes mellitus (DM) and atrial hypertension ( $\mathrm{HT})$, patients' improvement at 12 months after surgery, change in $\mathrm{BMI}(\triangle \mathrm{BMI})$ and percent excess BMI loss (\%EBMIL) [16]. Complications were defined as adverse events that occurred within 30 days of the procedure. Complications were classified according to "Standardized outcomes reporting in metabolic and bariatric surgery" by Brethauer 
et al. [16] Improvement was established as the reduction of medications or when the medications could be stopped [16].

\section{Matching}

The matching procedure was performed according to BMI $\left( \pm 1 \mathrm{~kg} / \mathrm{m}^{2}\right)$ and comorbidities (DM, HT). The controls were selected for each case using an algorithm described by Kawabata (1 : 1 matching procedure) [17]. Cases without a suitable control were excluded from the study.

\section{Statistical analysis}

Analysis was performed using SAS software, University Edition (SAS Institute Inc., Cary, NC, USA). Continuous outcomes were analyzed using the paired $t$-test, or Wilcoxon signed ranks test. Dichotomous outcomes were analyzed using McNemar's test or Fisher's exact test [18].

\section{Results}

A total of 178 patients (89 matched pairs) were included in the study. Table I shows the characteristics of the patients.

Length of stay tended to be longer in patients older than 60 years than in younger patients, but this was not significant $(p=0.10)$. There was also no significant difference in the complication rate between the 2 age groups $(p=0.59)$. Bleeding during a 30-day observation time occurred in 2 (2.24\%) older patients and 1 (1.1\%) younger patient. Leakage was diagnosed in 1 (1.1\%) younger patient and $3(3.4 \%)$ older patients (Table II).

Twenty-four (26.97\%) patients suffered from DM while 48 (53.93\%) had HT in both age groups. An improvement in HT was observed in $73.1 \%$ of patients older than 60 years and in $69.2 \%$ of patients younger than 40 years $(p=0.67)$. There was also improvement in DM in $40 \%$ of older patients and in $31.1 \%$ of younger patients ( $p=0.25$, Table III).

The $\triangle \mathrm{BMI}$ after 12, 24 and 36 months was significantly lower in patients older than 60 years. than in younger patients $(p=0.002, p=0.001$; $p=0.043$, respectively). Similarly, \%EBMIL after 12 , 24, and 60 months was significantly lower in patients older than 60 years than in younger patients ( $p=0.001, p=0.001, p=0.028$, respectively) (Table IV). Despite a lack of significance, the tendency for lower body weight loss in younger patients was maintained in subsequent follow-ups (Figure 3).

\section{Discussion}

In this study, older patients were matched with younger patients with the same demographics to evaluate whether age was significant for outcomes from SG. Both age groups achieved similar outcomes in terms of complications, LOS and improvement in comorbidities. Younger patients tended to have greater body weight loss compared with older patients at follow-up.

A meta-analysis showed that young patients had better weight loss than did elderly patients [11]. A similar outcome was found in our study. Neverthe-

Table I. Characteristics of the patients

\begin{tabular}{|lccc|}
\hline Characteristics & $\begin{array}{c}\text { Older } \\
\text { patients }\end{array}$ & $\begin{array}{c}\text { Younger } \\
\text { patients }\end{array}$ & P-value \\
\hline Age [years] & $\begin{array}{c}63.3 \pm 2.73 \\
(60-71)\end{array}$ & $\begin{array}{c}32.8 \pm 5.46 \\
(18-39)\end{array}$ & $<0.001$ \\
\hline BMI [kg/m²] & $46.1 \pm 6.1$ & $46.0 \pm 6.2$ & 0.2532 \\
\hline $\begin{array}{l}\text { Sex: } \\
\text { Women }\end{array}$ & $53(59.5 \%)$ & $51(57.3 \%)$ & \\
\hline Men & $36(40.5 \%)$ & $38(42.7 \%)$ & \\
\hline Comorbidities: & & & \\
\hline DM & $24(40.1 \%)$ & $24(40.1 \%)$ & \\
\hline HT & $48(81.4 \%)$ & $48(81.4 \%)$ & \\
\hline LOS [days] & $4.04 \pm 2.05$ & $3.63 \pm 1.24$ & 0.0985 \\
\hline
\end{tabular}

Values are mean \pm SD or $n(\%)$.

Table II. Complications in patients

\begin{tabular}{|lccc|}
\hline Complications & $\begin{array}{c}\text { Older } \\
\text { patients }\end{array}$ & $\begin{array}{c}\text { Younger } \\
\text { patients }\end{array}$ & $P$-value \\
\hline General & $8(8.9 \%)$ & $6(6.7 \%)$ & 0.5930 \\
\hline Leakage & $1(1.1 \%)$ & $3(3.4 \%)$ & 0.3173 \\
\hline Bleeding & $2(2.24 \%)$ & $1(1.1 \%)$ & 0.5637 \\
\hline
\end{tabular}

Table III. Improvement in comorbidities

\begin{tabular}{|lccc|}
\hline Comorbidities & $\begin{array}{c}\text { Older } \\
\text { patients }\end{array}$ & $\begin{array}{c}\text { Younger } \\
\text { patients }\end{array}$ & $P$-value \\
\hline Hypertension & $38(73.1 \%)$ & $36(69.2 \%)$ & 0.6698 \\
\hline $\begin{array}{l}\text { Diabetes } \\
\text { mellitus }\end{array}$ & $18(40 \%)$ & $14(31.1 \%)$ & 0.2482 \\
\hline
\end{tabular}


Table IV. Weight loss

\begin{tabular}{|c|c|c|c|}
\hline Parameter & $\begin{array}{c}\text { Older } \\
\text { patients }\end{array}$ & $\begin{array}{l}\text { Younger } \\
\text { patients }\end{array}$ & $P$-value \\
\hline \multicolumn{4}{|l|}{12 months: } \\
\hline$n$ & 89 & 89 & \\
\hline $\mathrm{BMI}$ & $35.4 \pm 6.5$ & $32.0 \pm 5.3$ & \\
\hline$\Delta \mathrm{BMI}$ & $10.6 \pm 4.4$ & $13.0 \pm 5.1$ & 0.0015 \\
\hline \%EBMIL & $53.9 \pm 24.5$ & $64.2 \pm 22.5$ & 0.0014 \\
\hline \multicolumn{4}{|l|}{24 months: } \\
\hline$n$ & 46 & 62 & \\
\hline $\mathrm{BMI}$ & $35.2 \pm 7.1$ & $30.4 \pm 4.4$ & \\
\hline$\Delta \mathrm{BMI}$ & $11.3 \pm 5.8$ & $15.7 \pm 5.6$ & 0.0002 \\
\hline \%EBMIL & $55.7 \pm 28.6$ & $75.5 \pm 19.5$ & 0.0003 \\
\hline \multicolumn{4}{|l|}{36 months: } \\
\hline$n$ & 16 & 21 & \\
\hline $\mathrm{BMI}$ & $37.5 \pm 9.3$ & $32.8 \pm 3.3$ & \\
\hline$\Delta \mathrm{BMI}$ & $8.1 \pm 5.5$ & $13.4 \pm 4.2$ & 0.0425 \\
\hline \%EBMIL & $46.1 \pm 37.3$ & $62.8 \pm 17.2$ & 0.064 \\
\hline \multicolumn{4}{|l|}{48 months: } \\
\hline$n$ & 8 & 8 & \\
\hline $\mathrm{BMI}$ & $40.1 \pm 10.9$ & $32.9 \pm 3.4$ & \\
\hline$\Delta \mathrm{BMI}$ & $6.7 \pm 8.2$ & $14.1 \pm 5.8$ & 0.0843 \\
\hline \%EBMIL & $34.1 \pm 35.0$ & $62.4 \pm 17.9$ & 0.0647 \\
\hline \multicolumn{4}{|l|}{60 months: } \\
\hline$n$ & 8 & 8 & \\
\hline $\mathrm{BMI}$ & $40.2 \pm 11.5$ & $30.5 \pm 2.9$ & \\
\hline$\Delta \mathrm{BMI}$ & $6.5 \pm 8.9$ & $16.5 \pm 6.1$ & 0.0509 \\
\hline$\%$ EBMIL & $33.3 \pm 38.0$ & $72.9 \pm 17.3$ & 0.0276 \\
\hline \multicolumn{4}{|l|}{72 months: } \\
\hline$n$ & 7 & 6 & \\
\hline $\mathrm{BMI}$ & $38.7 \pm 10.7$ & $28.8 \pm 1.7$ & \\
\hline$\Delta \mathrm{BMI}$ & $8.0 \pm 7.5$ & $17.9 \pm 5.2$ & 0.3125 \\
\hline \%EBMIL & $41.1 \pm 36.8$ & $81.2 \pm 9.2$ & 0.2672 \\
\hline \multicolumn{4}{|l|}{84 months: } \\
\hline$n$ & 3 & 5 & \\
\hline $\mathrm{BMI}$ & $43.7 \pm 14.9$ & $27 \pm 2.2$ & \\
\hline$\Delta \mathrm{BMI}$ & $6.3 \pm 10.3$ & $20.4 \pm 5.7$ & 1.000 \\
\hline \%EBMIL & $31.2 \pm 49.8$ & $89.8 \pm 10.6$ & 1.0 \\
\hline
\end{tabular}

Values are mean $\pm S D$ or $n$.

less, SG is still beneficial in older patients. The total body water volume decreases in older people, as well as muscle fibers, which are associated with sarcope-

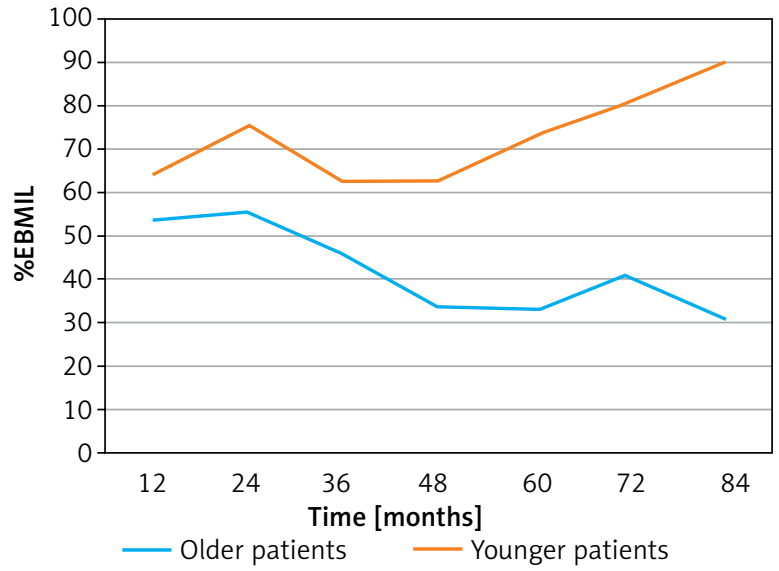

Figure 3. \%EBMIL during follow-up

nia and frailty syndrome [19]. The majority of patients in our study were women. Ochner et al. found that menopause-associated changes may reduce postoperative results after bariatric surgery [20]. We have also found the reason for a smaller weight loss effect in the patients' motivation for the surgery. Younger patients are more concerned about a better appearance than resolution of comorbidities after surgery. Therefore, younger patients are more motivated to lose weight, but not for medical reasons [21]. Moreover, in older people, energy expenditure is lower and life is more static than in younger people [22].

The study did not show any significant improvement in comorbidities between the groups, as previously reported [11]. Improvement, defined as withdrawal of medications or at least a reduction in consumed medicine, was observed in approximately $70 \%$ of patients with HT and more than one third of patients with DM. Similar results were found in recent randomized controlled trials [23, 24]. Older age may not be a negative factor for resolution of comorbidities. Reduction of medications can influence the quality of life. Even a decrease in taking drugs is a goal for successful surgery.

Furthermore, the complication rate was similar between the two age groups in our study. Despite the small sample size and matching procedure, our results may be generalized to the entire population, because similar findings were reported by other studies $[25,26]$. Importantly, especially among elderly people, perioperative care appears to play a crucial role in improving postoperative outcomes. Recent developments in perioperative care and introduction of enhanced recovery after surgery (ERAS) protocols in 
bariatric surgery and other surgical disciplines have led to faster recovery and reduction in postoperative morbidity [27-29]. The ERAS protocols have enabled elimination of some of the traditional risk factors for postoperative complications and prolonged length of hospital stay. Together with laparoscopic surgery, ERAS protocols are particularly beneficial in older patients [30-32]. However, most of the evidence for ERAS protocols is from other surgical disciplines, and this has not been confirmed for bariatric surgery. However, considering the pathophysiological background of ERAS protocols, these benefits should also be applicable to elderly obese patients [33].

Goals of surgery in the elderly population should include not only weight loss, but also improvement in physiological function and quality of life. Despite the physiological aspects not being analyzed in the study, bariatric surgery improves patients' quality of life and it is not related to weight loss [34, 35]. Further studies on this issue among elderly patients need to be performed.

Limitations of this analysis include the retrospective design of the study and the small sample size. According to the current Polish guidelines for bariatric surgery [36], procedures are recommended for patients aged 18-60 years, and those older than this age should be specially considered. The long-term follow-up results of patients older than 60 years who have bariatric surgery are unknown. There are the supposed reasons for the small sample size. Younger patients were carefully matched in our study so that the effect of age alone was examined. Our outcomes appear to overlap with similar previous studies [9-14]. Therefore our finding may be applicable to a larger population.

\section{Conclusions}

The study shows that patients older than 60 years benefit from bariatric surgery. Better weight loss is achieved in younger patients than in older patients, while maintaining a similar effect on the risk of complications and improvement in comorbidities. Therefore, sleeve gastrectomy should be considered as safe and effective in older people.

\section{Acknowledgments}

We thank Ellen Knapp, PhD, from Edanz Group (www.edanzediting.com/ac) for editing a draft of this manuscript.

\section{Conflict of interest}

The authors declare no conflict of interest.

\section{References}

1. WHO. Obesity and overweight. Fact sheet, June 2018.

2. WHO. Ageing and health. Fact sheet, January 2018

3. Cheng J, Gao J, Shuai X, et al. The comprehensive summary of surgical versus non-surgical treatment for obesity: a systematic review and meta-analysis of randomized controlled trials. Oncotarget 2016; 7: 39216-30.

4. Gloy VL, Briel M, Bhatt DL, et al. Bariatric surgery versus non-surgical treatment for obesity: a systematic review and meta-analysis of randomised controlled trials. BMJ 2013; 347: f5934.

5. Angrisani L, Santonicola A, lovino P, et al. Bariatric surgery worldwide 2013. Obes Surg 2015; 25: 1822-32.

6. Michalik M, Dowgiałto-Wnukiewicz N, Lech P, et al. Surgery of the elderly in emergency room mode. Is there a place for laparoscopy? Videosurgery Miniinv 2017; 12: 115-9.

7. Gebhart A, Young MT, Nguyen NT. Bariatric surgery in the elderly: 2009-2013. Surg Obes Relat Dis 2015; 11: 393-8.

8. Garofalo F, Denis R, Pescarus R. Long-term outcome after laparoscopic sleeve gastrectomy in patients over 65 years old: a retrospective analysis. Surg Obes Relat Dis 2017; 13: 1110-5.

9. Soto FC, Gari V, de la Garza JR, et al. Sleeve gastrectomy in the elderly: a safe and effective procedure with minimal morbidity and mortality. Obes Surg 2013; 23: 1445-9.

10. Ramirez A, Roy M, Hidalgo JE, et al. Outcomes of bariatric surgery in patients >70 years old. Surg Obes Relat Dis 2012; 8: 458-62.

11. Wang Y, Yi X, Li Q, et al. The effectiveness and safety of sleeve gastrectomy in the obese elderly patients: a systematic review and meta-analysis. Obes Surg 2016; 26: 3023-30.

12. Kaplan U, Penner S, Farrokhyar F, et al. Bariatric surgery in the elderly is associated with similar surgical risks and significant long-term health benefits. Obes Surg 2018 Mar 10 [Epub ahead of print]

13. Lainas P, Dammaro C, Gaillard M, et al. Safety and short-term outcomes of laparoscopic sleeve gastrectomy for patients over 65 years old with severe obesity. Surg Obes Relat Dis 2018; 14: 952-9.

14. Major P, Wysocki M, Janik M, et al. Impact of age on postoperative outcomes in bariatric surgery. Acta Chir Belg 2018; 118: 307-14.

15. Major P, Wysocki M, Pędziwiatr M, et al. More stapler firings increase the risk of perioperative morbidity after laparoscopic sleeve gastrectomy. Videosurgery Miniinv 2018; 13: 88-94.

16. Brethauer SA, Eisenberg D, Kim J, et al. Standardized outcomes reporting in metabolic and bariatric surgery. Surg Obes Relat Dis 2015; 11: 489-506.

17. Kawabata H, Tran M, Hines P. Using SAS to match cases for case control studies. Pap. 173-29. Bristol-Myers Squibb, Princeton, New Jersey; p. 1-7.

18. Breslow NE, Day NE, International Agency for Research on Cancer. Statistical methods in cancer research volume I-the analysis of case-control studies. Stat Methods Cancer Res 1980; 1: 346. 
19. Clegg A, Young J, lliffe S, et al. Frailty in elderly people. Lancet 2013; 381: 752-62.

20. Ochner CN, Teixeira J, Geary N, et al. Greater short-term weight loss in women 20-45 versus 55-65 years of age following bariatric surgery. Obes Surg 2013; 23: 1650-4.

21. Libeton M, Dixon JB, Laurie C, et al. Patient motivation for bariatric surgery: characteristics and impact on outcomes. Obes Surg 2004; 14: 392-8.

22. van Hout GC, Verschure SK, van Heck GL. Psychosocial predictors of success following bariatric surgery. Obes Surg 2005; 15 552-60.

23. Peterli R, Wölnerhanssen BK, Peters T, et al. Effect of laparoscopic sleeve gastrectomy vs laparoscopic roux-en-Y gastric bypass on weight loss in patients with morbid obesity: the SM-BOSS randomized clinical trial. JAMA 2018; 319: 255-65.

24. Salminen P, Helmiö M, Ovaska J, et al. Effect of laparoscopic sleeve gastrectomy vs laparoscopic roux-en-Y gastric bypass on weight loss at 5 years among patients with morbid obesity: the SLEEVEPASS randomized clinical trial. JAMA 2018; 319: 241-54.

25. Chang SH, Freeman NLB, Lee JA, et al. Early major complications after bariatric surgery in the USA, 2003-2014: a systematic review and meta-analysis. Obes Rev 2018; 19: 529-37.

26. Jędrzejewski E, Liszka M, Maciejewski M, et al. Age is not associated with increased surgical complications in patients after laparoscopic sleeve gastrectomy. Videosurgery Miniinv 2018; 13: 82-7.

27. Małczak P, Pisarska M, Piotr M, et al. Enhanced recovery after bariatric surgery: systematic review and meta-analysis. Obes Surg 2017; 27: 226-35.

28. Greco M, Capretti G, Beretta M, et al. Enhanced recovery program in colorectal surgery: a meta-analysis of randomized controlled trials. World J Surg 2014; 38: 1531-41.

29. Pisarska M, Małczak P, Major P, et al. Enhanced recovery after surgery protocol in oesophageal cancer surgery: systematic review and meta-analysis. PLoS One 2017; 12: e0174382.

30. Pędziwiatr M, Pisarska M, Kisielewski M, et al. ERAS protocol in laparoscopic surgery for colonic versus rectal carcinoma: are there differences in short-term outcomes? Med Oncol 2016; 33: 56.

31. Kisielewski M, Pędziwiatr M, Matłok M, et al. Enhanced recovery after colorectal surgery in elderly patients. Videosurgery Miniinv 2015; 10: 30-6.

32. Ljungqvist O, Hubner M. Enhanced recovery after surgery-ERAS - principles, practice and feasibility in the elderly. Aging Clin Exp Res 2018; 30: 249-52.

33. Pędziwiatr M, Mavrikis J, Witowski J, et al. Current status of enhanced recovery after surgery (ERAS) protocol in gastrointestinal surgery. Med Oncol 2018; 35: 95.

34. Major P, Matłok M, Pędziwiatr M, et al. Quality of life after bariatric surgery. Obes Surg 2015; 25: 1703-10.

35. Janik MR, Rogula T, Bielecka I, et al. Quality of life and bariatric surgery: cross-sectional study and analysis of factors influencing outcome. Obes Surg 2016; 26: 2849-55

36. Wyleżoł M, Paśnik K, Dąbrowiecki S, et al. Polish recommendation for bariatric surgery. Videosurgery Miniinv 2009; 4 Supp 1: 31-4.

Received: 26.10.2018, accepted: 27.11.2018. 